\title{
Linking the Public Acceptance of Wind Power to Technology Deployment
}

\section{Abstract}

Public acceptance and positive media image are the key features of any technology market deployment. This paper analyses the media image of wind power and the link between the media image of a technology, public acceptance and thus, successful market deployment. Indeed, the increase in wind power plants has been influenced by increased off-shore technology developments as well as subsidies and greater acceptance of the technology. This paper focuses on the latter by undertaking opinion mining approach with machine learning -based media analysis.

For the paper, media sentiment analysis of both editorial and social media was conducted using an M-Adaptive tool for media monitoring. The results indicate that the sentiment toward wind power is primarily positive in both editorial publications and social media (SoMe), and also at the project level of offshore projects. The role of SoMe seems to be increasing for public acceptance issues. When considering the effect of global climate negotiations, namely, Paris COP21, the United Nations conference on climate change, the effect on wind power was mostly negative in terms of the communication quantity and overall media sentiment.

Keywords: Public acceptance of technology; wind power; machine learning -based media analysis

\section{Introduction}

Global warming requires sound solutions to overcome its impacts and preserve global habitats for future generations still to come. The concerns over the anthropogenic carbon dioxide $\left(\mathrm{CO}_{2}\right)$ emission contribution to climate change are resulting in pressure to reduce atmospheric $\mathrm{CO}_{2}$ and climate agreements (e.g., the Kyoto Protocol, 1997; the Paris Agreement 2015). In response to the threat of climate change, governments worldwide are making commitments to reduce their reliance on fossil sources of energy and increase the use of low carbon energy sources, specifically nuclear and renewable energy (Devine-Wright, 2011). As a next step, solar power, wind farms, and technologies for carbon capture and storage are increasingly being deployed and further developed (Batel et al., 2013). At the end of 2014, more than 370 gigawatts of wind electricity were in operation worldwide, producing $5 \%$ of the world's electricity demand (IEA Wind 2014). The barriers to further 
commercialisation of the wind power market, however, are important, especially in China, and may hinder the $100 \mathrm{GW}$ capacity target by 2020. Thus, the diffusion of wind power is an important element for reducing greenhouse gas emissions as well as making worldwide progress in wind power technology (Liao et al., 2010). Lay attitudes toward technologies are essential, so the acceptance of technology is considered a critical item for the further widespread deployment of low-carbon technologies (Fleishman et al., 2010). Past examples suggest that public acceptance can be a key factor influencing deployment of new energy infrastructure cost-effectively, thereby affecting many technologies, such as CCS (Bradbury et al., 2009), nuclear power (Grove-White et al., 2006), Wind farms (Firestone and Kempton 2007), nanotechnology (Siegrist et al, 2007), gene technology (Siegrist, 2000), and many others. Public acceptance of such technologies can also be influenced by fears of radiation (Kim et al., 2013), $\mathrm{CO}_{2}$ being released from the ground, possibly causing suffocation (Wallquist et al., 2009), potential noise, or even threat to animals (Wolsink, 2007), or perhaps as yet unknown dangerous consequences (Zechendorf, 1994).

Lately, the environmental and societal problems related to energy use have influenced the development of sustainable energy technologies, such as windmills. Obviously, these projects face some opposition as a result (Wollsink, 2000). Hence, public acceptance of these technologies is crucial for their successful introduction into society (Huijts, et al, 2012). The steady rise in the number of publications on the topic indicates that public acceptance related to technologies continues to be the focus of much research attention. (Gupta et al., 2012). In general, surveys now implicate strong overall public support for wind power, while concrete projects have been seen to suffer from the "not in my backyard" (NIMBY) syndrome that some of the public hold (Wollsink, 2000).

In the case of renewable power technologies, such as wind power, public acceptance is recognised as being an important issue to resolve before shaping any widespread implementation of energy technologies. This issue also influences how the targets set by energy policy can be reached (DevineWright, 2007). Historically, many technologies have been associated with societal controversies and indeed then led to public rejection of their use; thus, it is important to understand the psychological determinants of positive societal acceptance of new emerging technologies (Gupta et al., 2012). With public acceptance having such a potential influence, studying the level of public acceptance becomes a necessity, as does in this case, the media image of wind power technology. The terms of such acceptance were studied by Batel et al. (2013) with the conclusion reached that there needs to be 
justification of a top down perspective while yet ignoring features like resistance, uncertainty, support, and apathy. (Batel et al., 2013).

According to these studies, a change in public attitudes is required if more radical and renewable energy technology deployment that is needed can actually happen (Devine-Wright, 2007). Barry et al. (2008) recognized that the main issues in transitioning to energy economy based on renewable energy will have only minor relations to technology, as such, and thus public acceptability of wind energy is the key. The acceptance cannot be taken for granted when wind energy moves from abstract or principled support by the public to actual local implementation. However, for renewable energy, robust and systematic research based on social science for public engagement will need to be urgently applied due to the targets set by several governments for that level of increased deployment. (DevineWright, 2012).

Recently, despite multiple studies concerning the acceptance of renewable power, the research has concerned mainly the techno-economic approach, with a minor focus on the social sciences (DevineWright, 2012). Most of the research is concentrated on land-based wind power with abundant literature existing on the public opinion, community involvement and the effects of projects on cultural landscapes. However, less research has focused on the nature of the public attitudes toward, for example, offshore wind power projects (Firestone et al., 2012). The same as for onshore developments, the public should be included in all decision-making regarding offshore wind farms, thus their having a key role should not be underestimated (Hagget, 2011).

Only limited factors have been included in many of the acceptance studies, thus there is an absence of comprehensive frameworks. There may have been a lack of technology acceptance related to the inclusion of that key factor (Huijts, et al., 2012). (Barry et al., 2008) describes NIMBY as follows: "Wind power development arouses strong opinions. For the general public, a high level of support nationally for wind power can be contrasted with opposition at the local level" Ek, (2005). This indicates there is generally a positive public opinion toward wind power; however, the support for wind power does seem to decrease with age and income. A study by Ladenburg (2008) also indicated that younger people are more positive toward wind power than are the older generations. The literature on public attitudes towards wind power indicates certain key assumptions as well, such as the opposition to wind power is considered to be deviant, its opponents ignorant, and the majority of the public does support wind power. Such trust is essential, so there is good reason to better understand the opposition so as to find ways to overcome it.(Aitken, 2009). 
It is to be noted as well that in addition to land-based wind farms, there has been much journalistic and editorial discussion on the opposition to offshore wind projects, and yet in-depth analysis of the factors underlying that resistance to, or even support for these developments has remained sparse. Further, there exist tendencies to generalize the opposition by using short-hand labels, such as 'NIMBY', and not examining the underlying reasons of that opposition (Firestone et al., 2012). The Oxford English Dictionary (OED), defines NIMBY as "A person who objects to the siting of something perceived as unpleasant or hazardous in their own neighbourhood, especially while raising no such objections to similar developments elsewhere" (OED 2018).

The research by Ladenburg (2008) on Danish windmills indicates, however, that people who are not living near the windmills have indicated more positive attitudes than those people living far away from the windmills. Yet, generally the Danish population seems to have a positive attitude, correlating with age (negatively), experience with off-shore wind farms, gender, and use of the beach. Kaldellis, (2005), indicated there is acceptance toward already built Greek wind parks, but resistance toward new ones. In terms of local project support, the divergence seems to be most strong when considering those people expecting to live in close proximity to the project (Aitken, 2010). An example of this syndrome is the Barendrecht onshore carbon dioxide storage site project in The Netherlands. The project was mainly delayed due to increased (local) opposition, influenced largely by shortcomings in the level of communication between the stakeholders and that (local) community. (Feenstra et al., 2010).

Sovacool et al. (2012) described the factors that form the basis of socio-political, community, and market acceptance of renewable electricity, including political commitment and a positive public image. The latter is the focus of this paper. If these factors are present, market acceptance does grow. However, in countries where these conditions are lacking, growth of installation is lacking (Sovacool et al., 2012). Jobert et al. (2007) implicated certain social acceptance-related factors that included ownership, visual impact, participation and information with implications directly attune to implementation, including the creation of a support network, local integration of the developer, and the access to ownership. Warren et al. (2009) indicated that some opposition concentrates on factual issues like intermittency, bird mortality, or job creation. Thus, the primary reasons for not reaching a consensus are mostly related to conflicting values, such as community (dis)empowerment and aesthetics, and these can be unquantifiable, perceptual, and shaped by the world view of a person. (Hall et al., 2013) investigated four common themes that have influenced such societal acceptance, namely, distributional justice, trust, procedural justice, and place attachment. 
Nevertheless, it should not simply be assumed that the opposition to wind power is illegitimate or that it can simply be dismissed as ignorant or misinformed. The literature must abandon the assumption that it knows who is 'right'. Instead, it must engage w the possibility that the objectors to wind power are not always 'wrong' (Aitken, 2010). People with an interest in environmental issues are, however, more likely to feel positive towards wind power than the average respondent will, and this viewpoint is affected by age (Ek, 2004). Ellis et al, (2007) reviewed the research on the opposition to wind farms and noted that previous criticisms had tended to provide descriptive rather than explanatory insights, and as a result, this research had not effectively informed the policy debate.

\section{Research Questions and Methodology}

2.1 Research Methodology: Big Data Utilization of Commercial Software, Computational Linguistics, Machine Learning, and Opinion Mining

The research methodology for this current study is based on utilising opinion mining via machine learning- based media-analysis, including a large number of editorial and social media sources. When compared to traditional news media that is capable of shaping public opinions on an issue by emphasising some elements of the broader controversy over others (Shah et al., 2002), Social Media offers more direct opinions including emotional content (Stieglitz and Dang-Xuan, 2013). The research principles presented in this paper have already been formerly applied in various fields of study, for example, public acceptance studies undertaken by traditional media analysis earlier smaller datasets. Further, sentiment analysis has previously been applied, for example in marketing research. Media frames have also been combined with cluster analysis and software-based sentiment analysis Bursher et al (2015). This study also applies social media along with the more traditional media for global and also local, analysis, while still attempting to retain an accurate and full comparability of datasets. Sentiment analysis/opinion mining as a method is related to a classification problem due to the prediction of polarity words like positive, negative and neutral (Kumar et al., 2017). Accuracy, data size, data sparsity, and sarcasm are considered the main uncertainty issues for sentiment mining classification and clustering, whereas sentiment classification mines written documents (comments, posts, reviews, tweets, etc.) about a product or service and classifies these documents into positive or negative opinion categories (Qiang Ye et al., 2014). Sentiment clustering groups the written documents (Kumar et al., 2017). Using opinion mining from big data, the quantity of analyzed media sources is increased significantly compared to traditional questionnaires and interviews or other 
means of media-analysis. However, big data does present new challenges for data processing, for example, -storage, -representation, pattern mining and visualization in those fields related to natural language processing, data mining, text mining, machine learning, social networks, and sentiment analysis (Bello-Orgaz et al., 2016). The unstructured data in social networking like blogs, reviews, posts, comments, and tweets are rapidly growing in their quantity and they represent a significant source to use as a basis for sentiment analysis (Zhang et al., 2008).

Machine learning tasks are typically classified into two broad categories, depending on whether there is a learning "signal" or "feedback" available to a learning system, namely, supervised and unsupervised learning. The majority of machine learning utilizes supervised learning wherein you have input variables (x) and an output variable (Y) and apply an algorithm to learn the precise mapping function from input to $t$ output. $Y=f(X)$ The goal is to approximate the mapping function so well that when you have new input data (x), you can predict the output variables (Y) for that data. Thus, is called supervised learning because the process whereby an algorithm learns from the training dataset can be thought of as a "teacher" who is supervising the learning process (Brownlee, 2016). This paper utilizes supervised learning, and it increases the hit accuracy from approximately $70 \%$ upwards to near $80 \%$.

Media framing, accompanied by cluster analysis and statistical methods, was not applied due to a comparison of editorial content to SoMe; thus dataset comparability became the key. However, in Table 1, certain key linkages are described at the European country level, wherein the media image has been compared to wind power deployment in each country. Furthermore, due to differences between the media framing between editorial media and SoMe, it was difficult to find suitable statistical methods for data-series analysis. This issue was a due to the fact that the main linkage was found to be first, between the quantity of media attention, and secondly, its quality. For example, large positive or negative attention has the most effect, and was supposed to be based on the literature as well. So having two effective variables combined into one and suggesting to make the difference, was challenging. Single datasets did not have any significant correlation one with the other.

The main benefits of choosing opinion mining via machine learning -based media analysis was the possibility of being able to utilise global vast datasets, including editorial content and SoMe, rapid processing of data and a smaller bias due to human perception and interpretation (Matthes and 
Kohring, 2008). Also, the significance of social media as a data provider has increased, making it a suitable platform for analysing public attitudes (Penalver-Martinez et al., 2014). In social media, different types of services exist. In Twitter, the messages or "tweets" are restricted to 140 characters; Facebook is a more social networking platform, Vkontakte is Facebook's Russian alternative, while blog writing offers possibilities of extended text creation and there deeper analysis. YouTube is a platform for posting videos, and Instagram one for posting pictures. Hence, these social media platforms have fundamental differences in how users can express themselves in a single message. Nevertheless, as this study has chosen to analyze media sentiment, the impact of these differences in expression should not be significant.

Given a big data -based, media-analysis, the amount of media hits to be analysed drastically increased, compared to more traditional news analysis or even interviews and questionnaires. Compared to traditional methods, the number of sources would be much smaller, and interview answers could vary depending on the participants and with the risk of receiving only socially desired responses (Sovacool and Ratan, 2012). The analysis in this paper thus researched the linkage from a public acceptance status of wind power technologies' to their possible connections to the recent increase in wind power technology market deployment globally.

The literature is reviewed to clarify the current social acceptance status of wind power, together with the media image and potential barriers for technology commercialisation, thereby opening that view to media-analysis. The literature findings were analysed and synthesised to obtain a clearer view of the effect of social acceptance on technology development, and how the current situation could be improved to help produce more widespread technology applications. Hence, the literature analysis became the basis for the media-analysis. The results are then generalised to further implicate and thus examine the general current status of the energy industry.

The practical application of the methodology was taken via a media-analysis of global editorial and SoMe sources, utilizing M-Adaptive software. In this study, the M-adaptive sources covered globally 3 million social media sites accompanied by 100,000 news outlets, 71 languages and 236 regions (MBrain, 2015). Sentiment analysis applied to this research applied machine learning aided by human to linguistic knowledge resulting in software offering suggestions of classifications, as estimated and corrected by humans. Further, the system self-improved its content classification, and divided it into sentiment categories. (M-Brain, 2015). The media-analysis was accomplished by inputting the keywords "wind power" to M-adaptive media monitoring software. The analysis was conducted for 
the time period July 22015 to July 2 2016, and included a total of 98,944 machine learning selected data points (see Figure 2) from both editorial publications $(37,106)$ and social media $(61,838)$. The analysis was then supplemented with half year data (996 hits) from selected active offshore wind projects between 1.12, 2016 and 25.5.2017. Figure 1 presents the quantity of wind power media hits across different media.

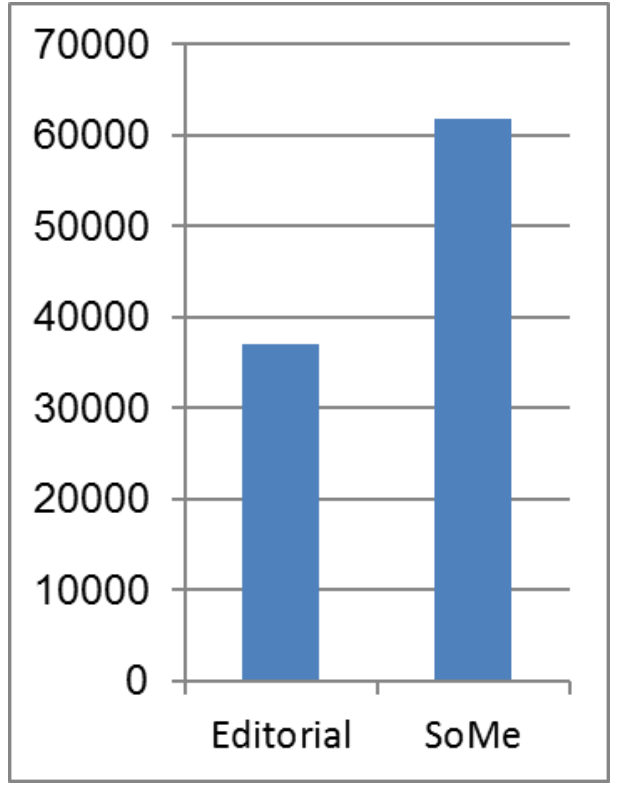

Figure 1. Media hits on wind power

In practice, during the media analysis, there was an automated classification to sentiment-coding expressions using five options: Negative, positive, mixed, neutral, and unknown, whereas the mixed classification contained conflicting elements- The opinions in the text were first recognised and classified into types automatically, including positive terms or quantifying expressions. For a given topic and a given document matching that topic, the quantity and sentiment class of that topic were then assessed and an overall compound judgement then displayed (M-Brain, 2015).

The method in question is definitely a new application in public acceptance research. However, opinion mining is currently a very active research focus area that mainly concentrates on natural language processing, computational linguistics and text analysis technologies. Thus, the target is to obtain $\mathrm{g}$ various added-value and informational elements from user opinions (Penalver-Martinez et al., 2014). The utilised methodology has a clear purpose for research. For example, it has been evident in online marketing, mainly in the manufacturing industry that relates to consumers, wherein manufacturers and distributors are faced with millions of discussion forum postings (Abrahams et al., 
2012). Due to the huge increase in online sources, such as discussion forums, blogs and such, there is a major need to develop precise computational tools to analyse people's preferences and attitudes (Neviaroyskaya et al., 2014).

Individuals and organisations are increasingly utilising public opinion in these media for their own key decision-making (Liu et al., 2012). To quantify opinions from, for example, product reviews, several systems have been created (Godpole et al., 2007). Capturing such general sentiment can be beneficial, although Nasukawa and Yi (2003) identified local sentiment as being more reliable than global document sentiment, due to human evaluators often failing to agree on the global sentiment of the same document (Godpole et al., 2007). In addition, it is also known now that text analysis by humans can contain biases; for instance, people often can pay greater attention to those opinions that are consistent with their own preferences (Liu et al., 2012). In order to improve the accuracy of sentiment analysis, it is important to properly identify the semantic relationships between the expressions of sentiment and the subject (Nasukawa et al., 2003). Sentiment analysis research is thus seen to be moving towards "content, concept, and context- based analysis of natural language text, supported by time efficient parsing techniques suitable for big social data analysis" (Cambria et al., 2013

Due to issues related to using commercial software, the accuracy of such a method is assessed by using M-Brain's internal tests, which show an average accuracy of $80 \%$ for one document. Errors are, however, possible with respect to any given individual document due to ambiguity, which is an inherent part of human language. It is a known fact, that humans are not agreeing $100 \%$ in such tests, as is the case for any artificial system, where humour, sarcasm and irony are beyond that system's abilities to understand fully. However, evaluating data trends is more accurate, and thus the amount of documents to be analysed has increased, as with large volumes of data, the overall model matches human judgment of the same data qualitatively. This aspect can be seen as the main issue, and it can influence the selecting method whenever media studies with large datasets are involved (M-Brain, 2015). Due to the large data-set herein compiled from global editorial and SoMe sources with the help of machine learning -based analytics, this method can generally be considered as very suitable for this type of analysis, although it is also noted, that a limitation for full accuracy does yet exist.

\subsection{Research Questions}


The findings of this study were carefully analysed in order to answer the research questions set forth for this study, together with their relevant implications. The research questions for this paper were (RQ1) what is the media image of wind power in both the editorial and the social media (SoMe), and (RQ2) how has this image affected the public acceptance and furthermore the deployment of that technology in the marketplace?

The complex chain from media image to technology market deployment in the case of wind power is presented in Figure 2, thus summarizing the impact of media image to actual technology market deployment.

Figure 2. Linkage of media-image to product market deployment of wind power

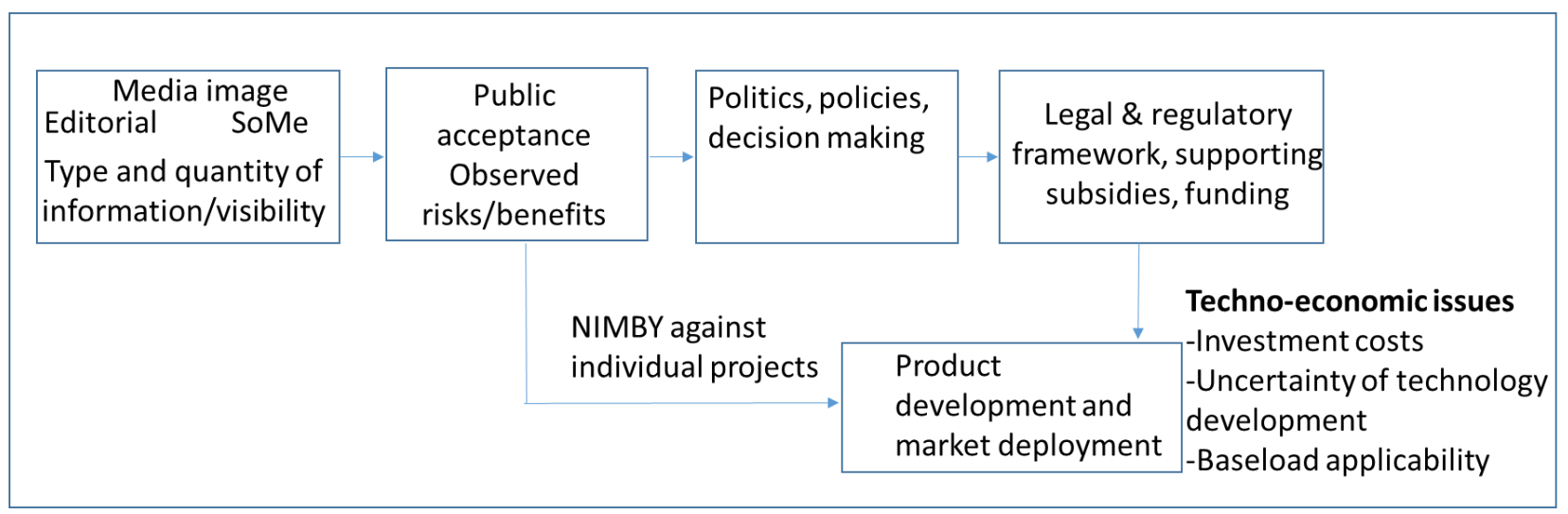

This chain starts with the media image, which influences o people's perceptions of technology. In addition to traditional news media, one that also shapes public opinion on a single topic via emphasizing one element over the other (Shah et al., 2002) is Social Media (SoMe), as it presents opinions directly from the user, together with stronger emotional input than gathered usually from the news media (Stieglitz and Dang-Xua 2013). The application of social media is also seen as supporting market intelligence and product development (Berendsen et al., 2015). Media framing has the potential to influence public acceptance, as the attention is focused and placed in a precise field of meaning (Heras-Saizarbitoria, et al., 2011).

Following this line of reasoning, in the PR-communication literature, the rule of effects describes the chain from media exposure via attention, comprehension, motivation, behavioural trial to actual sustained behavioural change (McCorkindale et al., 2013). According to this model, there exists the rule of halves/ describing, wherein the effect is halved in each step, leaving the percentage from media 
exposure to sustained behavioural change at $0.78 \%$, thus emphasising the need for extensive positive media exposure, if positive support of market deployment is needed. Further still, a stakeholder salience model (Mitchell et al., 1997) can be considered to try to estimate the effects of power, legitimacy and urgency of user posts in SoMe.

\section{Media Image data Analysis}

\subsection{The Global Media Image}

The large amount of data points obtained during the global media analysis provided a good basis for the sentiment analysis. The amount of data points was adequate to base a further analysis of media image/sentiment. In Figure 3 below, the sentiment toward wind power is depicted both for the editorial publications and the social media data hits.

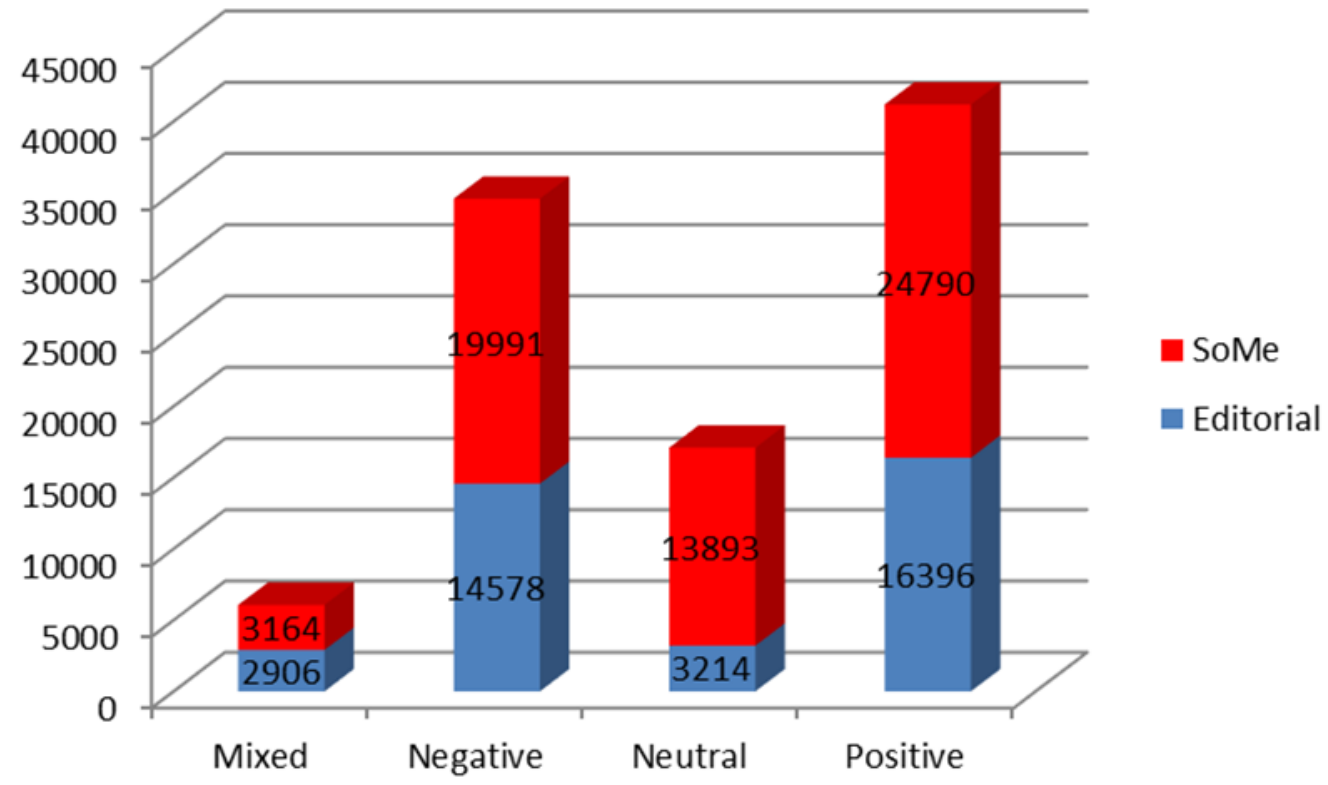

Figure 3. The sentiment analysis of SoMe compared to that of editorial publications

It can be seen in Figure 3 that for wind power, the results were mostly positive for both the editorial and the social media. Compared to, for example, the media attention for biomass power during about the same timeframe (Nuortimo et al., 2017), the level of hits was much larger, suggesting that wind power has been well presented in the media. The results also show that the wind power produced 
more positive hits in social media and also in the editorial publications, but with the ratio being almost two times more in the SoMe content, namely, 24,790 compared to 16,396 hits for the editorial content. The neutral hits were divided into a ratio of 3,214 in editorial content, and 13,893 in SoMe, with mixed hits, those which include conflicting elements. Thus, 3,164 hits were considered as mixed in SoMe, whereas the editorial content that number was 2,906. The negative hits, important due to their effectiveness, were concentrated in the social media with 19,991 compared to 14,578 found in the editorial context.

The ratio between the negative hits in SoMe is proportionally almost the same as those from the editorial publications. Further still, in the editorial publications, there were almost the same amount of negative hits as there were positive, while the emphasis on positive media image results came mainly from the social media. This result indicates a generally positive public attitude, due also to the high number of neutral hits, which indicating an extended exposure of the topic on the social media channels. Figure 4 demonstrates that $44 \%$ of the editorial exposure was positive with neutral content over $50 \%$. The number of mixed (8\%) and neutral $(9 \%)$ hits was relatively small, indicating a consensus for a viewpoint favouring wind power. Overall, this result can be seen as a positive indication of the general public acceptance of wind power in the editorial publications. The percentage of negative hits in the editorial publications (39\%) was larger than the share of negative hits in the social media (32\%), indicating slightly more negative attitudes toward wind power than seen in SoMe.

\section{Editorial}

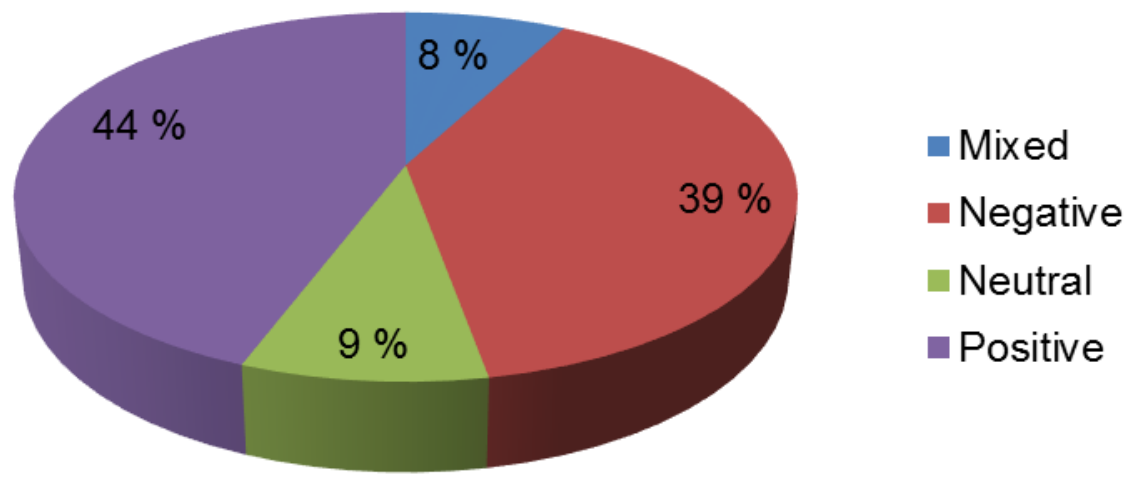

Figure 4. Sentiment analysis of the editorial publications 
From Figure 5 below, it is also evident that public sentiment toward wind power in social media was mostly positive with a $40 \%$ share, compared to $32 \%$ negative hits, while when taken together with the neutral hits $(23 \%)$, the share is over $60 \%$. However, the relative large amount of neutral hits indicates that some groups have yet to decide their attitudes, which can also be considered as an indication of the need to increase the communication efforts in SoMe. Generally, this distribution of hits can be seen as a strong indicator of a positive attitude existing among the laypeople and thus a strong public acceptance.

\section{SoMe}

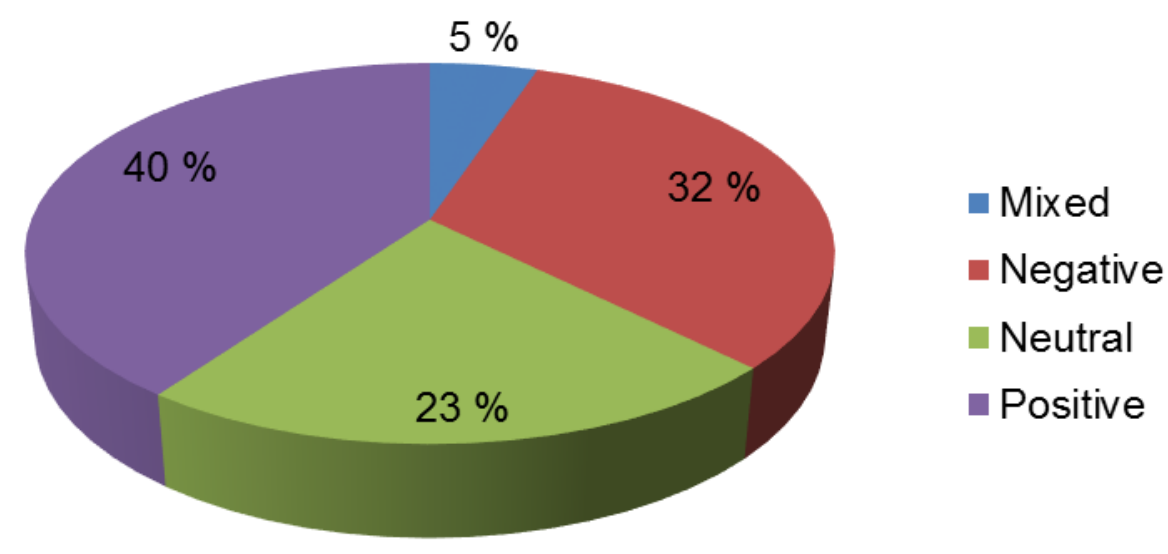

Figure 5. Media sentiment toward wind power found in Social Media

Figure 6 illustrates the very important aspect of public acceptance, namely, the social media sentiment toward wind power across different media. Dividing the social media sentiment by type of media reveals that blog writing attracted most of the social media attention with 25,796 hits, wherein the largest share was positive at 11,508 hits, and negative opinion was almost the same with 11,162 hits. Also, the number of neutral (750) and mixed $(2,376)$ hits held only a minor share of the total, compared to the positive and negative. This result can be considered as an indication of polarity in the views. Twitter was active with 22,816 mostly neutral hits $(11,822)$, and 6,810 positive, 81 mixed and 4,101 negative. When compared to Facebook (1,245), Google Plus (5,204), Forums (2,243), YouTube (2,326), VKontakte (227), Instagram (536) and Tumblr (1,473), the twitter and blog writing seemed to be the most influential of all the SoMe sources. Due to a more visible number of positive 
hits, the social media effect can also be considered as being quite large when the public positive opinion towards technology was formed. This result is mainly due to the possibility of individuals' expressing their opinions directly without need for objectivity, such as would be the case in the editorial media.

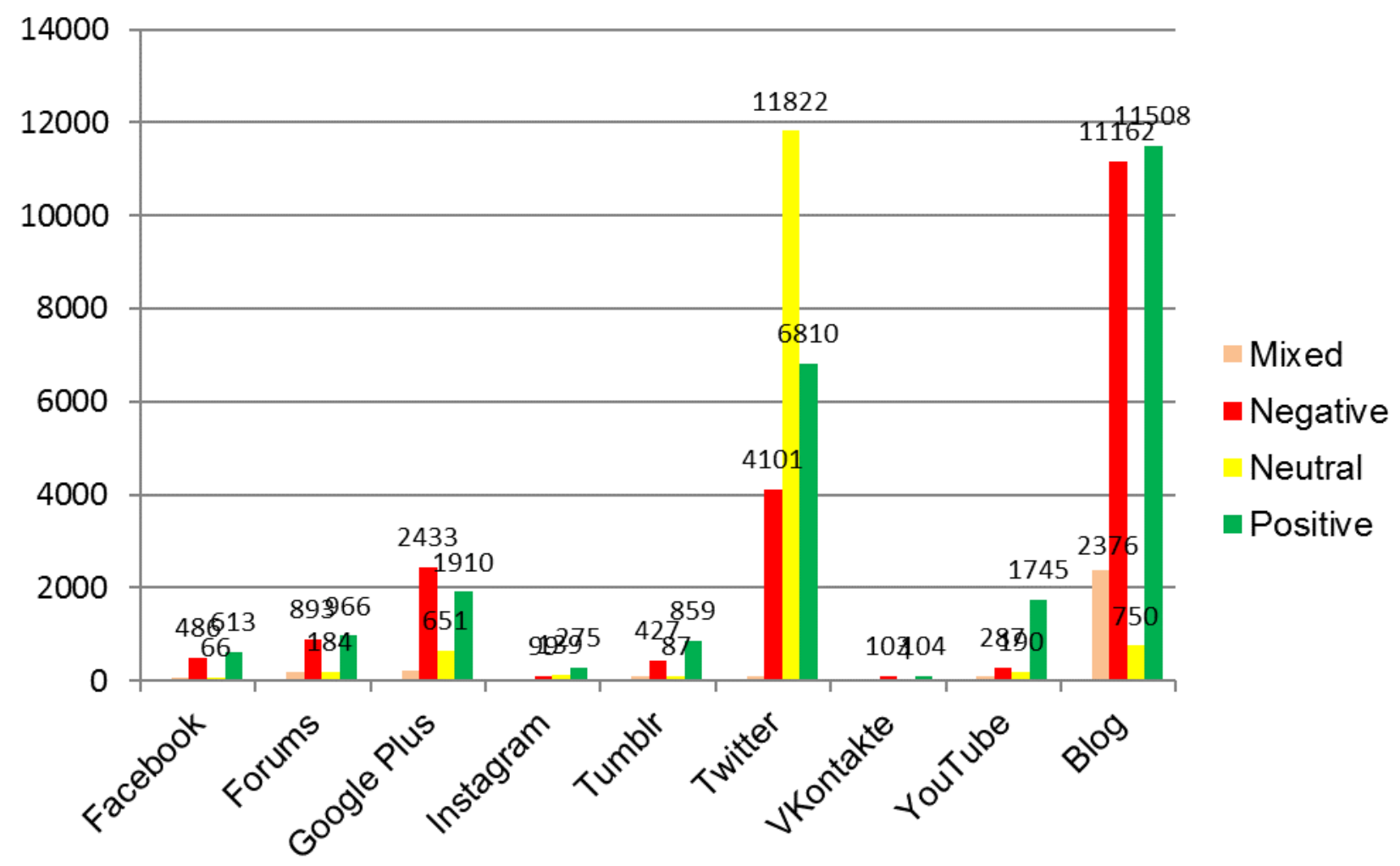

Figure 6. Expression of the social media sentiment analysis by media type

On the global level, the gender differences are noted in Figures 7 and 8 with female sentiment being slightly more positive than male sentiment at only a $7 \%$ difference. 


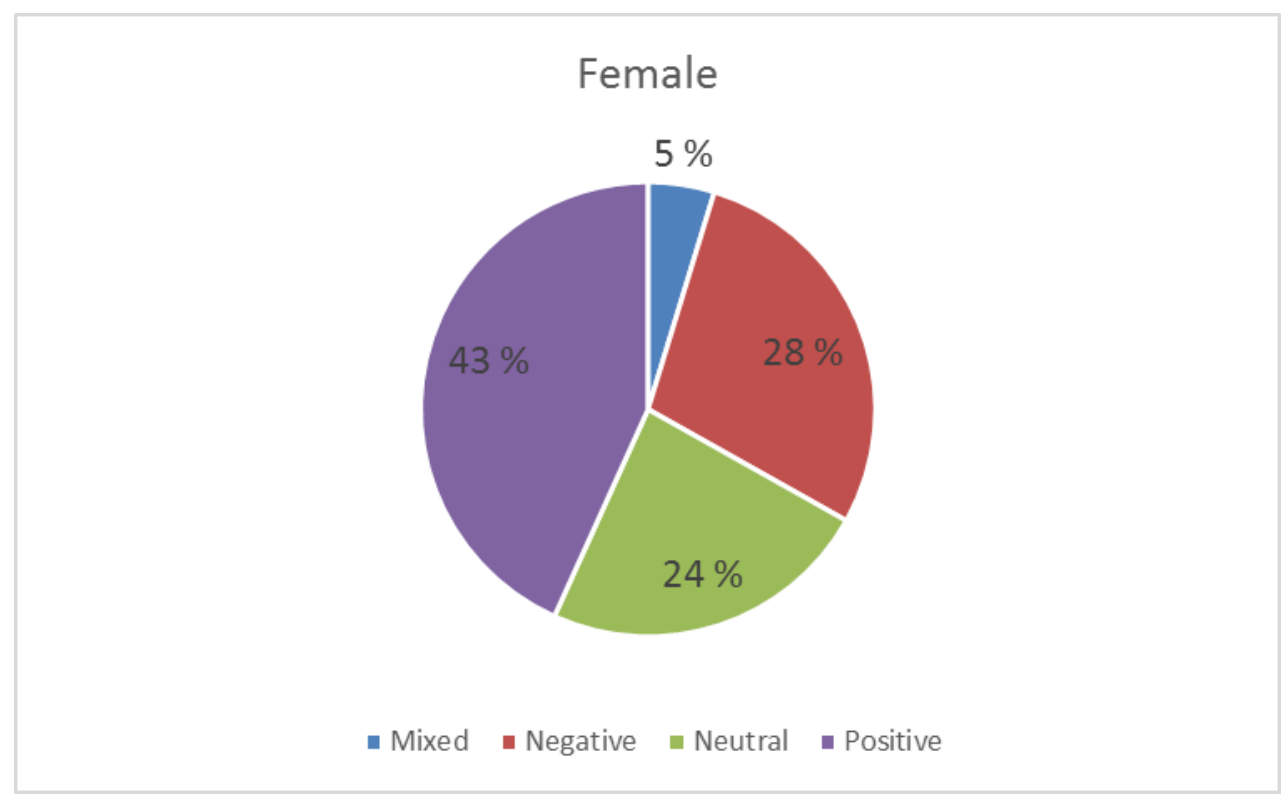

Figure 7. Female media sentiment toward wind power

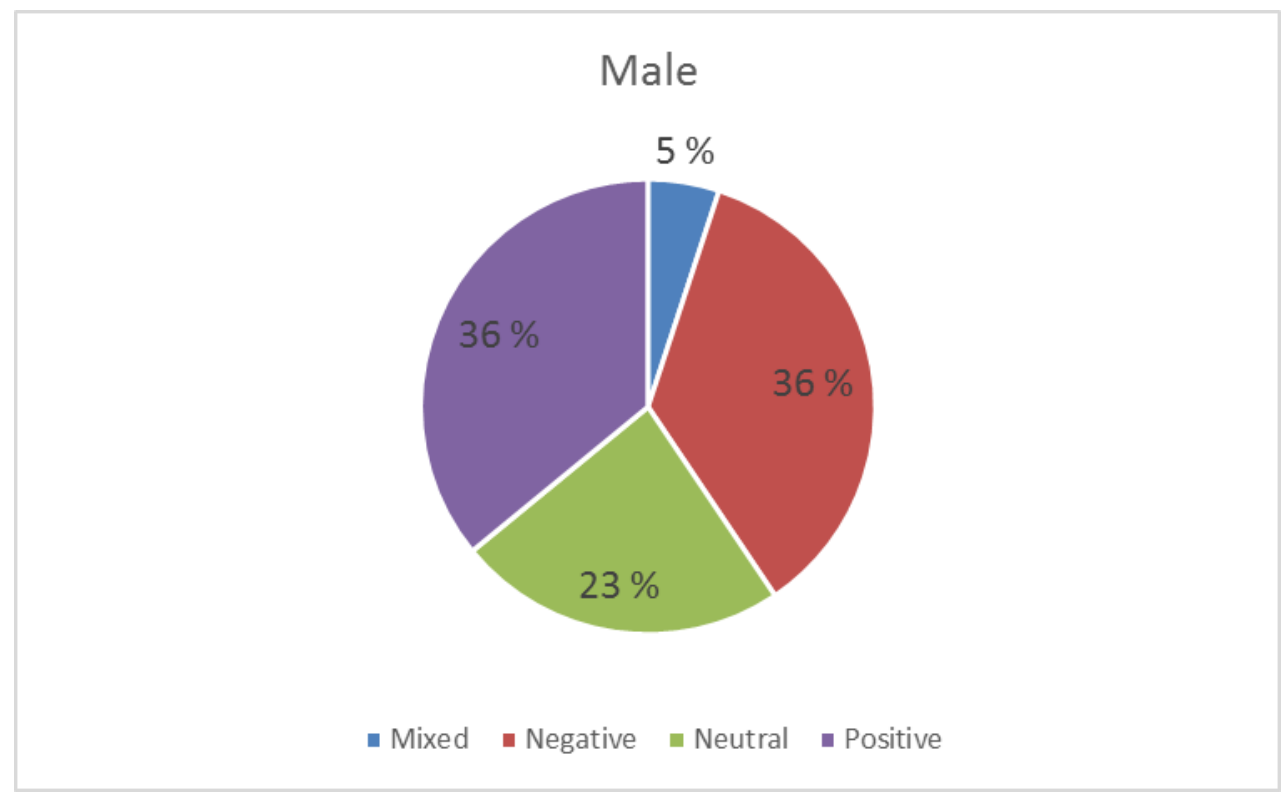

Figure 8. Male media sentiment toward wind power

\section{Media Image During the Paris COP Negotiations}

During the Paris COP negotiations that took place between November 30, 2015 and December 11, 2015 there were a total of 1,263 hits, and during that period the relative media attention paid to wind power was approximately more than half of the total media compared to a regular average month with 5,121 hits (calculated as a monthly average). The sentiment was found to be mostly positive; 
however, there were more negative views compared to a regular average month. During the Paris COP21, the deviation of media sentiment changed (see Figure 9). Proportionally, the number of negative hits increased during that period. The total number of negative hits was 512, largely concentrated on SoMe (280). The positive content contained a total of 208 editorial hits and 724 hits in social media, thus indicating a greater than monthly average negative content. Due to the low number of mixed hits (73), it appeared that the media attention during Paris COP21 was indeed more polarised.

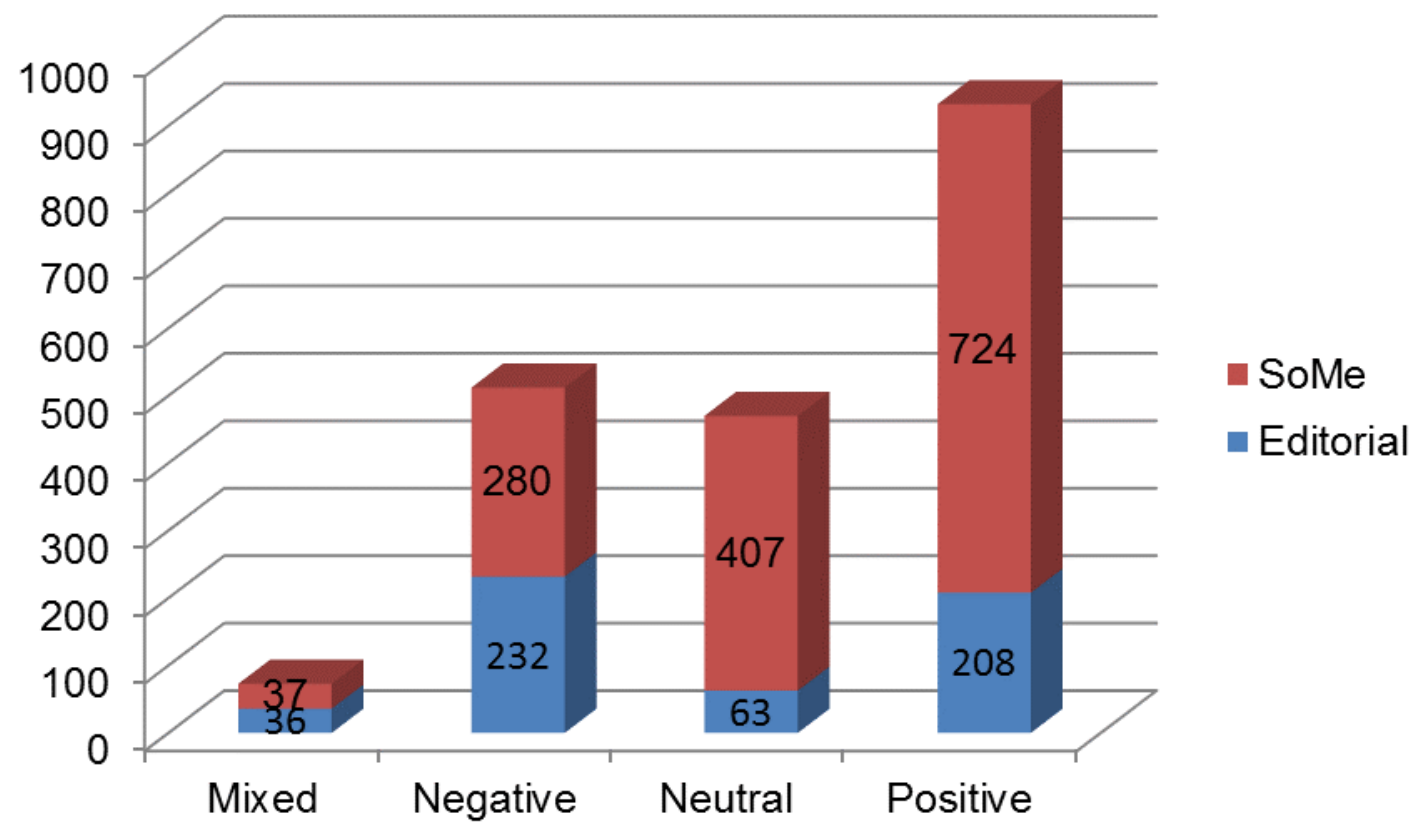

Figure 9. Sentiment analysis of wind power for SoMe vs. editorial publications during Paris COP time period

The media hits during the global climate negotiations also indicated some change in the media sentiment toward wind power. The media sentiment seemed to be more negative than normally with $43 \%$ compared to the $39 \%$ monthly average. At the same time, the social media attitude was also a bit more negative than normal with a $39 \%$ share (se Figure 10) of hits compared to $31 \%$ as the monthly average. 


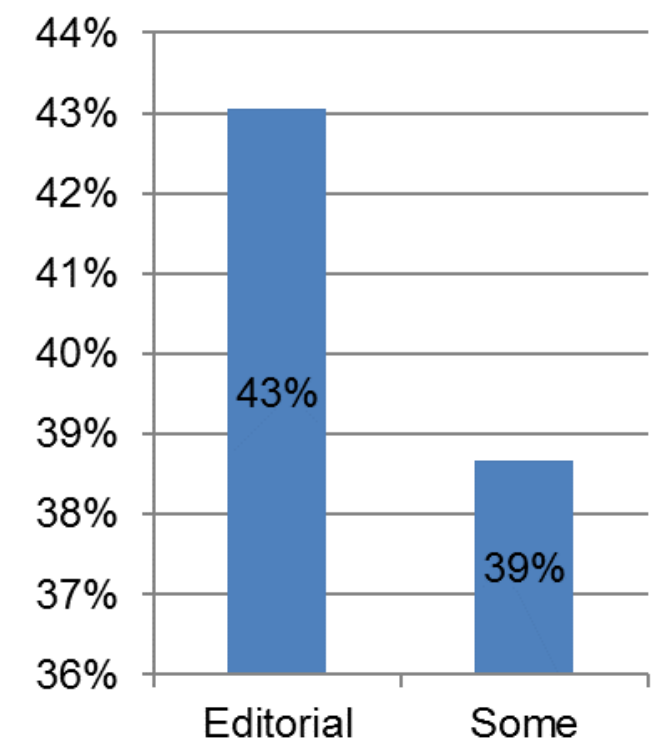

Figure 10. The effects of the Paris COP negotiation on media exposure

\section{Media image on a continental/country level for an indication of deployment impact}

When moving from global media sentiment to a more local level, several differences appear. Figure 11 notes the media sentiment in different world regions. It seems that the largest negative sentiment was the US, compared to Europe, for example, with more than half of the total hits, but generally more positive ones. 


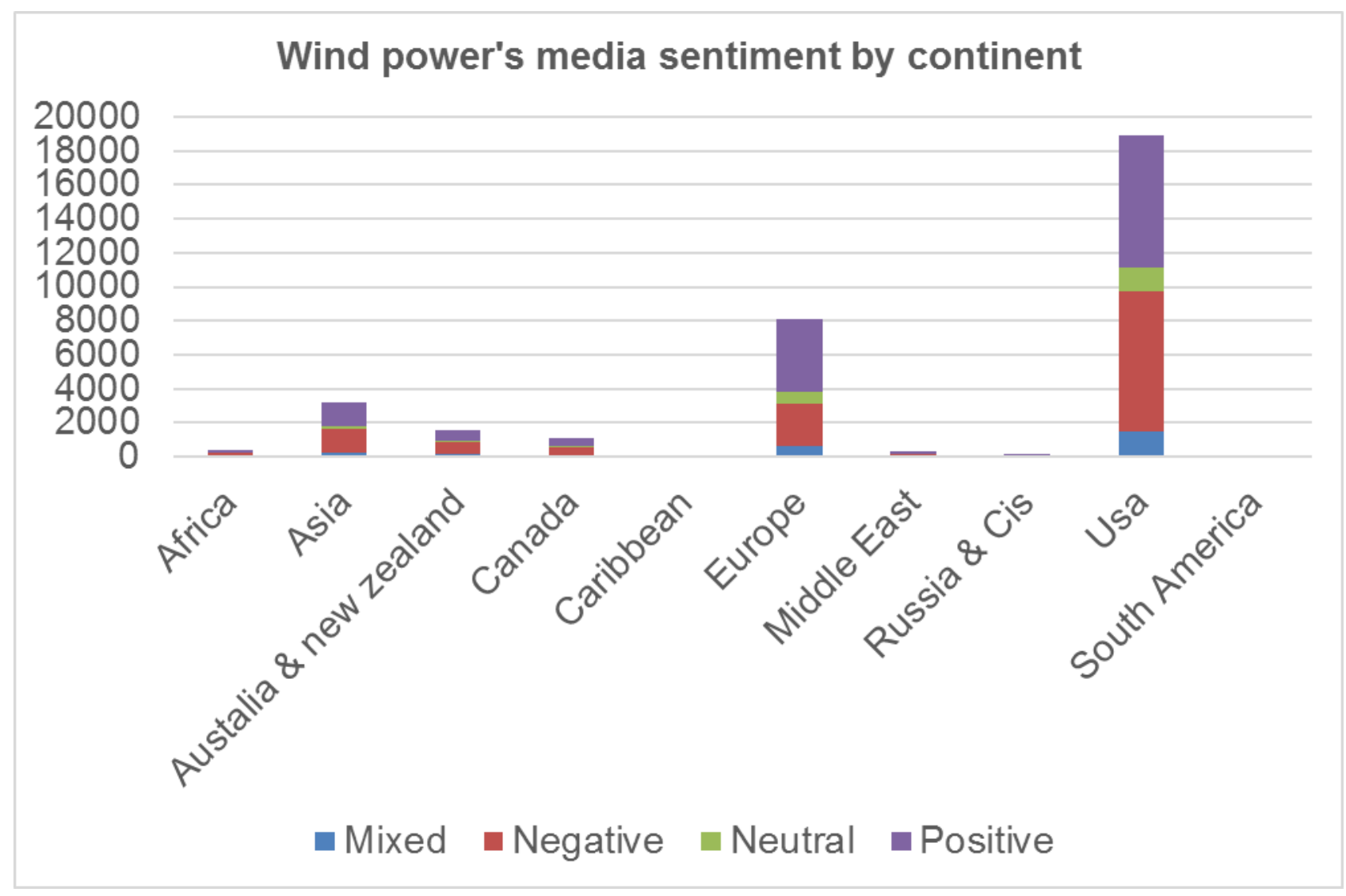

Figure 11. Media sentiment in different world regions by continent

When moving to the country level in Europe, the sentiment clearly became more diversified. Table 1 summarizes the media sentiment differences in the European countries. 
Table 1:Media sentiment effect on deployment of wind power in European countries

\begin{tabular}{|c|c|c|c|c|c|c|c|c|c|c|}
\hline & $\begin{array}{c}\text { Installed } \\
\text { capacity, end } \\
2015 \text { (MWe) }\end{array}$ & $\begin{array}{l}\text { Added wind } \\
\text { capacity } 2016 \\
\text { (MWe) }\end{array}$ & $\begin{array}{c}\text { Added } \\
\text { wind } \\
\text { capacity } \% \\
\text { of installed } \\
\text { base }\end{array}$ & Tot.Hits & $\begin{array}{c}\text { Negative } \% \\
\text { media } \\
\text { attention }\end{array}$ & $\begin{array}{c}\text { Media impact } \\
\text { (quantity/quality) }\end{array}$ & $\begin{array}{l}\text { Position } \\
\text { in } \\
\text { installed } \\
\text { capacity }\end{array}$ & $\begin{array}{l}\text { Position } \\
\text { in wind } \\
\text { power } \\
\text { increase } \\
2016\end{array}$ & $\begin{array}{c}\text { Pos. in } \\
\text { negativ } \\
\text { e media- } \\
\text { attentio } \\
\quad n\end{array}$ & $\begin{array}{l}\text { Growth } \\
\text { rate } \\
\text { order }\end{array}$ \\
\hline Germany & 44941 & 5443 & $12 \%$ & 769 & $11 \%$ & Large, positive & 1 & 1 & 13 & 6 \\
\hline France & 10505 & 1561 & $15 \%$ & 108 & $26 \%$ & Small, medium & 4 & 2 & 6 & 4 \\
\hline Turkey & 4694 & 1387 & $30 \%$ & 152 & $93 \%$ & Small, negative & 7 & 3 & 1 & 1 \\
\hline Netherlands & 3443 & 887 & $26 \%$ & 62 & $24 \%$ & Small, medium & 11 & 4 & 8 & 2 \\
\hline UK & 13809 & 736 & $5 \%$ & 4825 & $37 \%$ & Large, negative & 3 & 5 & 4 & 10 \\
\hline Poland & 5100 & 682 & $13 \%$ & 9 & $22 \%$ & Small, medium & 8 & 6 & 9 & 5 \\
\hline Sweden & 6029 & 493 & $8 \%$ & 155 & $12 \%$ & Small, positive & 6 & 7 & 12 & 8 \\
\hline Ireland & 2446 & 384 & $16 \%$ & 313 & $55 \%$ & Medium, negative & 13 & 8 & 2 & 3 \\
\hline Italy & 8975 & 282 & $3 \%$ & 326 & $21 \%$ & Medium, medium & 5 & 9 & 10 & 13 \\
\hline Portugal & 5050 & 268 & $5 \%$ & 8 & $0 \%$ & Small, positive & 9 & 10 & 15 & 11 \\
\hline Austria & 2404 & 228 & $9 \%$ & 38 & $11 \%$ & Small, positive & 14 & 11 & 14 & 7 \\
\hline Denmark & 5064 & 220 & $4 \%$ & 326 & $26 \%$ & Medium, medium & 10 & 12 & 7 & 12 \\
\hline Belgium & 2218 & 177 & $8 \%$ & 37 & $32 \%$ & Small, medium & 15 & 13 & 5 & 9 \\
\hline Romania & 2976 & 52 & $2 \%$ & 20 & $40 \%$ & Medium, negative & 12 & 14 & 3 & 14 \\
\hline Spain & 23025 & 49 & $0 \%$ & 192 & $14 \%$ & Small, positive & 2 & 15 & 11 & 15 \\
\hline
\end{tabular}

In Table 1 above, the statistics on deployment and the annual increase of wind power from GWEC are compared to the media-image in the respective country. From the table it is clearly visible that Germany had large positive media-attention, which may have impacted deployment also. France had a small quantity of media hits together with medium quality of media-attention and with second largest yearly increase in wind power capacity. The largest annual growth was in Turkey, together with small negative media attention and no foreseen effect on deployment. In the UK, large negative attention may have stalled wind power deployment; yet although UK has the third largest installed capacity, it still is tenth in wind power growth rate order. So deployment may be stalling. Most of the countries with a low growth rate order (Spain, Austria, Portugal) had fairly small positive media-image, negatives also included: Romania (medium/negative), Denmark(medium, medium) and Italy(medium, medium). This result is mostly in line with the theory basis, wherein the quantity of media attention is an important factor in positive development of public acceptance and also deployment of the technology.

To test the theory basis for the NIMBY -effect relation to media attention (see Figure 12), the media image of three offshore projects was analysed. Due to these projects not being land-based, NIMBY should be small or non-existing, and if the link from acceptance to deployment also exists, also positive.

\section{Media Image Level of Offshore Projects}

To study the NIMBY effect on project level, the media image of thee offshore wind farms currently in active development was summarized, namely Wikinger (350 MWe) (Wikinger, 2017) and Nordsee One(Nordsee 
One, 2017); (332 MWe) offshore projects in Germany; and the Dunkirk offshore project (Dunkirk, 2017) in France (750MWe). These results are presented in Figures 12-13 below

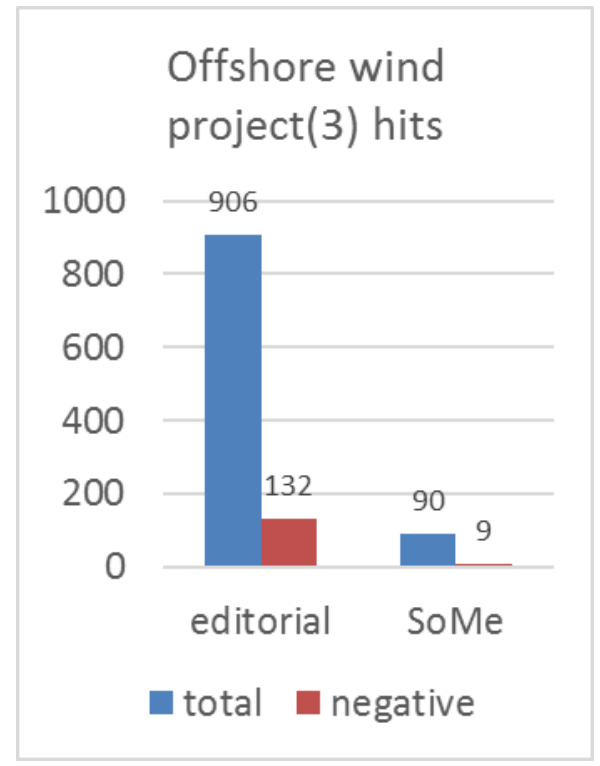

Figure 12. Media image of three offshore wind projects

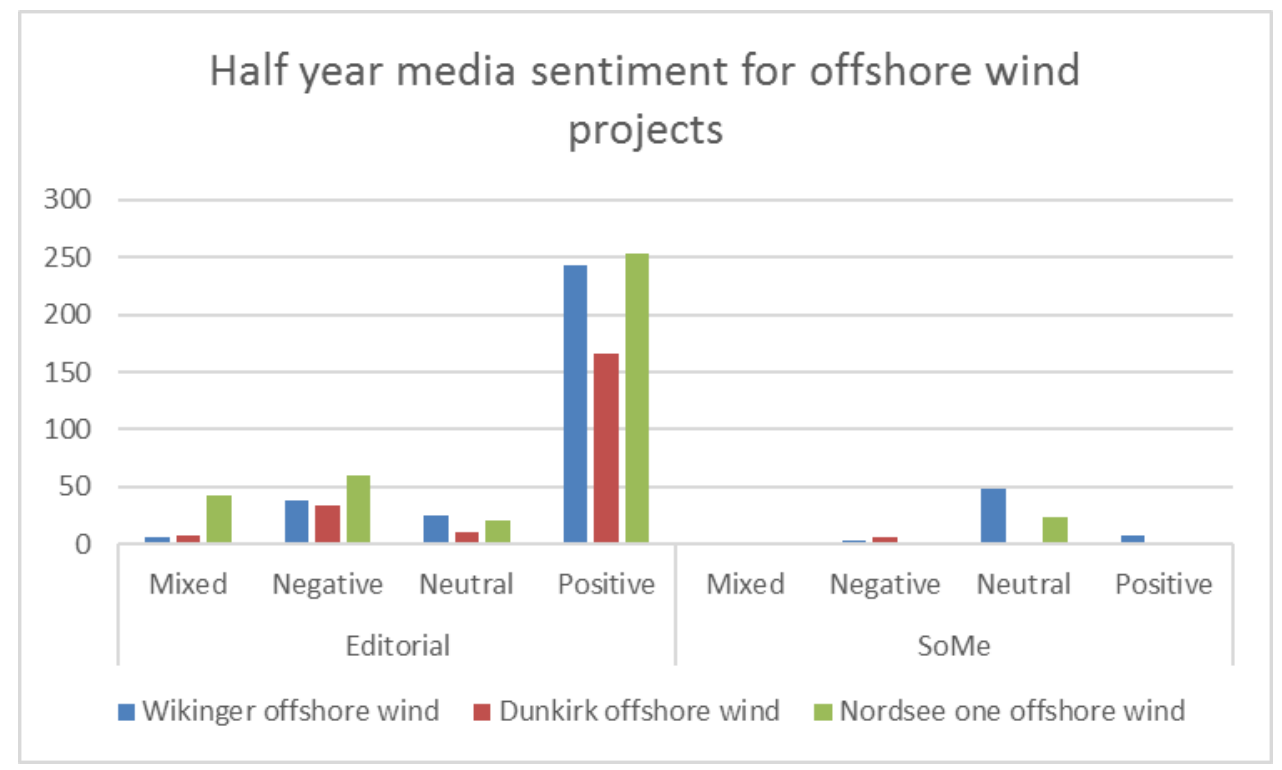

Figure 13. Media attention given three offshore wind projects currently under development

In Figures 12 and 13, it is clearly visible that three selected offshore wind projects have had a similar type of media sentiment, generally very positive. The main attention was concentrated in the editorial media. The small negative media attention given in SoMe is an indication of smaller NIMBY for offshore wind power. 


\section{Discussion}

In this media study, the search word of wind power was inputted into the M-adaptive software with a time range of July 2, 2015 to July 2, 2016. This analysis was supplemented with an analysis of three offshore projects during the time December 1, 2016 to May 25, 2017. The total media sentiment toward wind power was found to be generally positive during the analysis period due to the large amount of positive and neutral SoMe hits. For the offshore wind projects, the sentiment was also positive, while the small amount of negative hits in SoMe indicated very low NIMBY for the offshore wind projects. The media attention thus was correlated well for both project level and country level.

In the editorial media, the sentiment seemed to be a bit more negative. This study indicates that general public opinion is an important factor for social acceptance and is derived for that aspect, as well for political decision- making, as, for example, for Twitter where there were over 11,000 neutral tweets concerning wind power during the period analysed. When considering the effect of international events, such as the global climate negotiations of Paris COP 21, the media sentiment seems to have been influenced by such events either one way or another. In this case, the effect on wind power was mostly negative, so thus with no further possible explanations than other issues potentially stealing the media attention, the media attention on wind power decreased temporarily.

The results from the country analysis indicate that the media image effect followed the rule of effects, which indicated the need for larger media exposure. Then, via a complex chain by first getting public acceptance, formulate local policies, legislation and legal and regulatory framework, the effect towards technology market deployment is possible. NIMBY, however, is a more straightforward phenomenon, and in the case of offshore wind power, it is a good example to use to prove that this type of methodology is valid, due to the positive media image for the three active offshore wind projects. This view agrees with the theoretical framework presented in Figure 1, where a project located outside your own backyard should get a smaller NIMBY, and the positive effect from a general positive image gets better all the way to project level with a larger effect. This result is also an indication of a trend, namely that offshore wind will indeed have increased deployment in the future.

The limitations of this study include the use of keywords, as the outcome might prove to be different with the use of different keywords. Furthermore, framing, cluster analysis, and other statistical methods were found to be challenging to apply, due to the comparison of large datasets for both the 
Editorial media and SoMe. However, there has been criticism of the identification of news frames using statistical methods in a way that would be truly conceptually valid. (Carragee and Roefs, 2004). This topic thus is one for further research. When compared to the qualitative methods presented by Sovacool and Ratan (2012), the advantages of research interviews include the facilitation of a wider flow of information; thus answers are not restricted to only responses and categories anticipated by the researcher. This aspect can be helpful when dealing with value laden or subjective issues.

The differences in how individuals express themselves in a single message in social media may also have a certain impact level on the obtained results. Furthermore, thorough content analysis was impossible due to a large dataset, even though the sentiment analysis was tested by humans via random testing, thereby agreeing with the approximate $80 \%$ accuracy provided by M-Brain. The factors that were underlying the current sentiment were also clearly not present when utilizing this type of method, only the outcomes.

The fact that regional differences were not thoroughly taken into account in this study is a key general point of concern for data validity when making generalisations. The factors contributing to sentiment in each country were not clearly visible. Although global warming and the resulting agreements are of a global nature, technology commercialisation in and of itself is subject to regional politics, legislation, and their support mechanisms, such as subsidies, especially true in the case of wind power, despite recent advancement in its economic performance. However, the main benefit of these results is the ability to observe global trends and development directions, and also highlight the differences in country and project levels. This study also highlights quite well the differences due to the various channels of communication in the wind power context. The general trend toward increasing public acceptance towards wind power, however, despite these issues, was still clearly visible here.

From the media-analysis, it is clear that at the global level, the visibility in media, contributing to public acceptance toward wind-power is increasing and seems to be a positive indicator of wind power deployment. The subject has attracted much attention in both editorial content and SoMe. Especially, social media is influencing wind power technology acceptance in a positive way. Further still, as a result of increasing acceptance, the editorial publications here had almost the same number of negative hits as positive one. The emphasis toward this positive media image can be seen as resulting mainly from social media and its media contributions. One conclusion from this finding is that the role of social media has been increasing compared to that of traditional editorial publications. Also, a link to European wind power subsidies and other support mechanisms can be precisely 
established, due to the public support tending to influence political decision- making, and via that route, also national support mechanisms, such as subsidies.

The main methodological contribution of this study is its presentation of an example of the current state of art marketing research applications in a study on the public acceptance of wind power. Therefore, this study does offer a new view for questionnaires, or interview -based studies with moderate datasets and hundreds of data points, as applied by e.g., Heras-Saizarbitoria et al., (2011). There also seems to be a future basis for longitudinal data-series analyses, a feature that has been somewhat missing in the previous studies.

As a scientific implication, this study agrees with the findings of Devine-Wright, (2012), Sovacool et al. (2012), and Hagget, (2011) by highlighting how social acceptance is an important factor for wind power deployment, and should be included as an essential part of any technology deployment studies. Further, this study emphasises the importance of a technology media image and especially the social media image for public acceptance issues. However, methods for how to influence the media image positively are not discussed in this study. In terms of the NIMBY issues presented by Wollsink (2000) and Firestone et al. (2012), this study contributes further views of offshore wind projects. The main contribution is incorporating a method, formerly utilised mainly for marketing purposes, to wind power public acceptance studies, thereby bringing a new angle and focus to wind power- related media and social acceptance issues. This approach is a more modern one when compared to several interview, or questionnaire-based studies, which can include datasets consisting of some hundred data points are used in most of the earlier similar types of research.

The managerial implications relate also to method development. This paper utilises a method for its media image analysis on wind power, namely, a big data machine learning- based large dataset sentiment analysis. It is a method that has not been used to any large extent for this purpose earlier. Due to the development of technology, these methods are estimated to be increasingly relevant in the future. The method can be applied to general large dataset sentiment analysis and its results can be beneficial for managers, currently or in the future, who are considering the planning and implementing a wind power project. This paper documents the need to tackle public engagement, and stresses the need for social media participation, in the case of such project developments and any indication of local or global public resistance. By using this kind of approach, the points of most resistance can be identified and targeted with further actions, for example, more positive PR, and further, the results from such actions cant hen be monitored in real time. 
As a policy implication, this method may be suitable for taking public acceptance into account whenever creating legal frameworks for power production subsidies. It would offer a real- time view of public acceptance of different power production technologies as well as single such projects, thus with advantage of observing changes in project opposition.

\section{Conclusion and Recommendations for Future Research}

In this study, the wind power media image was found to be globally positive with certain variations in country and project level. From the country level analysis, the main indications were that a larger positive media image can speed up technology deployment, while a large negative image can slow it down, whereas smaller exposure, whether it is positive or negative, will clearly have less of an impact on such deployment. A positive image of offshore wind projects offers some indication of further future deployment due to its high level of acceptance, and low level of NIMBY. In the case of wind power, the media-image effect on technology deployment was emphasised, due the wind power market being highly subsidy driven, and subsidies always clearly affected via local politics.

The scientific contributions of this paper are twofold. First, it introduces a big-data, machine learning -based application to use for media study, which was then validated for a public acceptance example case, namely wind power. The main benefit of this method is its rapid response time, large reach in data, and accuracy in that human biases are not involved. Results are in-line with the literature, and the data series correlate with each other like it was expected based on the literature, thereby partly validating the accuracy of the methodology used here. This method also allows for rapid measurement of data quantity and quality, thus making possible a comparison of different datasets and an estimate of the effect of media exposure to eventual actual project deployment. The accuracy of machinebased analysis was also tested randomly.

Secondly, the study highlights the complex chain that runs from media-image via public acceptance and politics to actual market deployment, and studied that chain at the global, continental, country and local project level. This focus is definitely a fairly new angle to use for public acceptance research and indeed is grounds for increased emphasis on the general public, now ever more present via SoMe for possible stakeholder theories, such as the stakeholder salience model (Mitchell et al., 1997).

Based on this study, public acceptance seems to be an essential aspect of increasing the utilisation of wind power, and due to less NIMBY in offshore wind farms, it also seems to drive the market in that direction. Based on the media analysis, wind power seems to have a positive media image on the 
global level, and also for offshore projects, especially in social media, and media is seen as having a positive effect on further technology deployment. Machine learning -based, media-analysis indicates that the amount of popular positive communication is increasing constantly in social media, potentially benefitting the public acceptance of wind power technologies.

As a method implication, the media image of technologies' affecting larger audience groups' public acceptance can now be studied using machine learning -based media analysis. This type of analysis identifies the majority of attitudes in both editorial publications and the social media, thereby providing a faster way for legislators, scientists, and policy makers to gather information on public attitudes. This tool provides new grounds also for political decision- making. Due to the ever increasing amount of data available globally, both from editorial and SoMe sources, this method can be considered as the modern way to summarise large data sets that would otherwise be difficult, if not impossible, to handle. When compared to human- based analysis, the method is also superior, as reading tens of thousands articles takes enormous amounts of time, and the results often come too late for appropriate or viable decision- making. Hence, despite the evident inaccuracies, when single data points are observed and when large data sets are compared to each other, machine learning based analysis is indeed a method more suitable for that purpose.

The media image of wind power was found to be positive, implicating there is a need to push new regulations and policies to speed up wind power development. The amount of negative media attention was a bit larger during the Paris COP 21 meeting, implying the potential impacts of relevant large events and the appearance in the media. Policies' favouring wind power can be created as an implication of positive media image, however, not in my backyard. Those attitudes still need to be overcome, and they should be addressed locally, with the help of more qualitative approach.

The answer to the research questions in this paper, based on this study, entails realizing that the media image of wind power being positive in both editorial and social media and the public acceptance and technology market deployment being relatively positive. However, when considering the effect of global climate negotiations, namely Paris COP, the effect on wind power was mostly negative, indicating that other issues and technologies may have stolen the media attention. The media attention also varied at the country level, with countries having larger media exposure and a seemingly larger effect on deployment as well. The main correlation herein was mostly between the quantity of media attention and the sentiments, by following the rule of effects (McCorkindale et al., 2013).

The potential regional or local resistance toward wind power projects was also addressed in this study. These are matters that are also referred to as NIMBY syndrome (Wollsink, 2000), yet leaving room 
for further studies. For this purpose, more detailed data analysis is still needed with a specific focus on the communication about local land- based projects, possibly with the help of media framing, interviews etc. In addition, the further classification of respondents was inadequate in this particular study, leaving no room for analysing the results related to age as offered by Ek (2005) and Ladenburg (2008). In further research, both methodology development for the utilization of opinion mining as well as a specific approach that adds more qualitative data, such as questionnaires and interviews, to this type of larger media analysis, can prove to be most interesting. 


\section{References}

Abrahams, A.S., Jiao, J., Fan, W., Wang, G.A. and Zhang, Z. (2013) 'What's buzzing in the blizzard of buzz? Automotive component isolation in social media postings', Decision Support Systems, Vol. 55, No. 4, p.871.

Aitken, M. (2009) 'Wind power planning controversies and the construction of "expert" and "lay" knowledges', Science as Culture, Vol. 18, No. 1, pp.47-64.

Aitken, M. (2010) 'Why we still don't understand the social aspects of wind power: a critique of key assumptions within the literature', Energy Policy, Vol. 38, No. 4, pp.1834-1841.

Barry, J., Ellis, G. and Robinson, C. (2008) 'Cool rationalities and hot air: a rhetorical approach to understanding debates on renewable energy', Global Environmental Politics, Vol. 8, No. 2, pp.67-98.

Batel, S., Devine-Wright, P. and Tangeland, T. (2013) 'Social acceptance of low carbon energy and associated infrastructures: a critical discussion’, Energy Policy, Vol. 58, pp.1-5.

Bello-Orgaz, G., Jung, J.J. and Camacho, D. (2016) 'Social big data: recent achievements and new challenges', Information Fusion, Vol. 28, pp.45-59.

Berendsen, G., Middel, R., Pieters, I., Angard, F. and Hillerström, F. (2015) 'Social media within sustainable product development: an exploratory multiple case study on the perception of social media usability in the new product development process', Int. J. Technology

Intelligence and Planning, Vol. 10, No. 3/4, pp.273-293.

Bradbury, J., Ray, I., Peterson, T., Wade, S., Wong-Parodi, G. and Feldpausch, A. (2009) 'The role of social factors in shaping public perceptions of CCS: results of multi-state focus group interviews in the US', Energy Procedia, Vol. 1, No. 1, pp.4665-4672.

Brownlee, J. (March 16, 2016) [online] 'Machine Learning Algorithms: Supervised and Unsupervised Machine Learning Algorithms', https://machinelearningmastery.com /supervised-and-unsupervised-machine-learning-algorithms/ (accessed 18 February 2018).

Burscher, B., Vliegenthart, R. and de Vreese, C. (2015) 'Frames beyond words: applying cluster and sentiment analysis to news coverage of the nuclear power issue', Social Science Computer Review, 1-16.

Cambria, E., Schuller, B., Xia, Y. and Havasi, C. (2013) 'New avenues in opinion mining and sentiment analysis', IEEE Intelligent Systems, Vol. 28, No. 2, pp.15-21.

Carragee, K.M. and Roefs, W. (2004) 'The neglect of power in recent framing research', Journal of Communication, Vol. 54, No. 2, pp.214-233.

COP21 (2015) Paris Agreement, United Nations Framework Convention on Climate Change, Paris, France, 2015.

Devine-Wright, P. (2007) 'Reconsidering public attitudes and public acceptance of renewable energy technologies: a critical review', Manchester: School of Environment and Development, University of Manchester, UK.

Devine-Wright, P. (2011) Renewable Energy and the Public From NIMBY to Participation. Earthscan, Routledge, Oxon, UK, ISBN: 978-1-84407-863-9. 
Dunkirk wind farm [online] http://www.offshorewind.biz/2017/05/16/france-pre-selects-10dunkerque-offshore-wind-bidders/ (accessed 16 June 2017).

Ek, K. (2005) 'Public and private attitudes towards "green" electricity: the case of Swedish wind power', Energy Policy, Vol. 33, No. 13, pp.1677-1689.

Ellis, G., Barry, J. and Robinson, C. (2007) 'Many ways to say "no", different ways to say "yes": Applying Q-methodology to understand public acceptance of wind farm proposals', Journal of Environmental Planning and Management, Vol. 50, No. 4, pp.517-551.

Feenstra, C.F.J., Mikunda, T. and Brunsting, S. (2010) [online] 'What happened in Barendrecht? Case study on the planned onshore carbon dioxide storage in Barendrecht, the Netherlands', Global CCS Institute, Commonwealth Scientific and Industrial Research Organisation (CSIRO). https://www.globalccsinstitute.com/publications/what-happened-barendrecht (accessed 15 September 2017).

Fleishman, L.A., De Bruin, W.B. and Morgan, M.G. (2010) 'Informed public preferences for electricity portfolios with CCS and other low - carbon technologies', Risk Analysis, Vol. 30, No. 9, pp.1399-1410.

Firestone, J. and Kempton, W. (2007) 'Public opinion about large offshore wind power: underlying factors', Energy Policy, Vol. 35, No. 3, pp.1584-1598.

Firestone, J., Kempton, W., Lilley, M.B. and Samoteskul, K. (2012) 'Public acceptance of offshore wind power across regions and through time", Journal of Environmental Planning and Management, Vol. 55, No. 10, pp.1369-1386.

Godbole, N., Srinivasaiah, M. and Skiena, S. (2007) 'Large-scale sentiment analysis for news and blogs', ICWSM, Vol. 7, No. 21, pp.219-222.

Grove-White, R., Kearnes, M., Macnaghten, P. and Wynne, B. (2006) 'Nuclear futures: assessing public attitudes to new nuclear power’, Political Quarterly, Vol. 77, No. 2, pp.238-246.

Gupta, N., Fischer, A.R.H. and Frewer, L.J. (2012) 'Socio-psychological determinants of public acceptance of technologies: a review', Public Understanding of Science, Vol. 21, No. 7, pp.782-795.

Hall, N., Ashworth, P. and Devine-Wright, P. (2013) 'Societal acceptance of wind farms: analysis of four common themes across Australian case studies', Energy Policy, Vol. 58, pp.200208.

Haggett, C. (2011) 'Understanding public responses to offshore wind power', Energy Policy, Vol. 39, No. 2, pp.503-510.

Heras-Saizarbitoria, I., Cilleruelo, E. and Zamanillo, I. (2011) 'Public acceptance of renewables and the media: an analysis of the Spanish PV solar experience', Renewable and Sustainable Energy Reviews, Vol. 15, No. 9, pp.4685-4696.

Huijts, N.M.A., Molin, E.J.E. and Steg, L. (2012) 'Psychological factors influencing sustainable energy technology acceptance: a review-based comprehensive framework', Renewable and Sustainable Energy Reviews, Vol. 16, No. 1, pp.525-531. 
IEA WIND (2014) Annual Report. [online]

https://www.ieawind.org/annual_reports_PDF/2014/2014\%20AR_smallfile.pdfIEA, 2014 (accessed 3 February 2016).

Jobert, A., Laborgne, P. and Mimler, S. (2007) 'Local acceptance of wind energy: factors of success identified in French and German case studies', Energy Policy, Vol. 35, No. 5, pp.27512760 .

Kaldellis, J. (2005) 'Social attitude towards wind energy applications in Greece', Energy Policy, Vol. 33, No. 5, pp.595-602.

Kim, Y., Kim, M. and Kim, W. (2013) 'Effect of the Fukushima nuclear disaster on global public acceptance of nuclear energy', Energy Policy, Vol. 61, pp.822-828.

Kumar A.J., Abirami S. and Trueman T.E. (2017) 'Sentiment mining approaches for big data classification and clustering.' In a book Seetha, Hari, Murty, M. Narasimha, Tripathy, B. K. (Eds); Modern Technologies for Big Data Classification and Clustering. EBSCO Publishing: eBook Collection (EBSCOhost) - printed on 9 November 2017 4:39 AM.

Kyoto Protocol, New York: United Nations, 1997.

Ladenburg, J. (2008) 'Attitudes towards on-land and offshore wind power development in Denmark; choice of development strategy', Renewable Energy, Vol. 33, No. 1, pp.111-118.

Liao, C., Jochem, E., Zhang, Y. and Farida, N.R. (2010) 'Wind power development and policies in China', Renewable Energy, Vol. 35, No. 9, pp.1879-1886.

Liu, B. and Zhang, L. (2012) 'A survey of opinion mining and sentiment analysis', Mining Text Data, pp.415-463, Springer.

McCorkindale, T., DiStaso, M.W. and Carroll, C. (2013) 'The power of social media and its influence on corporate reputation', The Handbook of Communication and Corporate Reputation, pp.497-512.

Matthes, J. and Kohring, M. (2008) 'The content analysis of media frames: toward improving reliability and validity', Journal of Communication, Vol. 58, pp.258-279.

M-Brain (2015) [online] https://www.m-brain.com/ Corporate communications. Received 11/2015.

Mitchell, R.K., Agle, B.R. and Wood, D.J. (1997) 'Toward a theory of stakeholder identification and salience: defining the principle of who and what really counts', Academy of Management Review, Vol. 22, No. 4, pp.853-886.

Nasukawa, T. and Yi, J. (2003) 'Sentiment analysis: capturing favorability using natural language processing', Proceedings of the 2nd International Conference on Knowledge Capture, pp.70-77.

Neviarouskaya, A., Aono, M., Predinger, H. and Ishizuka, M. (2014) 'Intelligent interface for textual attitude analysis', ACM Transactions, Vol. 5, No. 3, Article 48.

Nordsee one wind farm (2017) [online] http://www.4coffshore.com/windfarms/nordsee-onegermany-de28.html (accessed 16 June 2017)

Nuortimo, K., Härkönen, J. and Karvonen, E. (2017) 'Exploring the social acceptance of biomass power', Interdisciplinary Environmental Review, Vol. 18, No. 1, pp.14-27. 
Oxford English Dictionary (2016) [online] http://www.oed.com/ (accessed 10 March 2016).

Peñalver-Martinez, I., Garcia-Sanchez, F., Valencia-Garcia, R., Rodríguez-García, M.Á., Moreno, V., Fraga, A. and Sánchez-Cervantes, J.L. (2014) 'Feature-based opinion mining through ontologies', Expert Systems with Applications, Vol. 41, No. 13), pp.5995-6008. doi:10.1016/j.eswa.2014.03.022.

Shah, D.V., Watts, M.D., Domke, D. and Fan, D.P. (2002) 'News framing and cueing of issue regimes: explaining Clinton's public approval in spite of scandal', Public Opinion Quarterly, Vol. 66, No. 3, pp.339-370.

Siegrist, M. (2000) 'The influence of trust and perceptions of risks and benefits on the acceptance of gene technology', Risk Analysis, Vol. 20, No. 2, pp.195-203.

Siegrist, M., Cousin, M.-E., Kastenholz, H. and Wiek, A. (2007) 'Public acceptance of nanotechnology foods and food packaging: the influence of affect and trust', Appetite, Vol. 49, No. 2, pp.459-466.

Stieglitz, S. and Dang-Xuan, L. (2013) 'Emotions and information diffusion in social mediasentiment of microblogs and sharing behavior', Journal of Management Information Systems, Vol. 29, No. 4, pp.217-248.

Sovacool, B. and Ratan, P.L. (2012) 'Conceptualizing the acceptance of wind and solar electricity', Renewable and Sustainable Energy Reviews, Vol. 16, No. 7, pp.5268-5279.

Wikinger wind farm [online] http://www.4coffshore.com/windfarms/wikinger-germany-de47.html (accessed 16 June 2017).

Wind statistics [online] http://www.gwec.net/global-figures/graphs/ (accessed 16 June 2017).

Wallquist, L., Visschers, V.H.M. and Siegrist, M. (2009) 'Lay concepts on CCS deployment in Switzerland based on qualitative interviews', International Journal of Greenhouse Gas Control, Vol. 3, No. 5, pp.652-657.

Wolsink, M. (2000) 'Wind power and the NIMBY-myth: institutional capacity and the limited significance of public support', Renewable Energy, Vol. 21, No. 1, pp.49-64.

Wolsink, M. (2007) 'Wind power implementation: the nature of public attitudes: equity and fairness instead of "backyard motives", Renewable and Sustainable Energy Reviews, Vol. 11, No. 6, pp.1188-1207.

Ye, Q., Li, H., Wang, Z. and Law, R. (2014) 'The influence of hotel price on perceived service quality and value in e-tourism: an empirical investigation based on online traveler reviews', Journal of Hospitality \& Tourism Research, Vol. 38, No. 1, pp.23-39.

Zechendorf, B. (1994) 'What the public thinks about biotechnology', Bio/Technology, Vol. 12, pp.870-875.

Zhang, C., Zuo, W., Peng, T. and He, F. (2008) 'Sentiment classification for Chinese reviews using machine learning methods based on string kernel', Third International Conference on Convergence and Hybrid Information Technology 11-13 November 2008, pp.909-914. 